\title{
On Augmented HEGY Tests for Seasonal Unit Roots*
}

\author{
Tomás del Barrio Castro \\ Department of Applied Economics \\ University of the Balearic Islands
}

\author{
Denise R. Osborn \\ Economics, School of Social Sciences \\ University of Manchester
}

\author{
A.M. Robert Taylor \\ School of Economics \\ University of Nottingham
}

September 2011

\begin{abstract}
In this paper we extend the large sample results provided for the augmented Dickey-Fuller test by Said and Dickey (1984) and Chang and Park (2002) to the case of the augmented seasonal unit root tests of Hylleberg et al. (1990) [HEGY], inter alia. Our analysis is performed under the same conditions on the innovations as in Chang and Park (2002), thereby allowing for general linear processes driven by (possibly conditionally heteroskedastic) martingale difference innovations. We show that the limiting null distributions of the $t$-statistics for unit roots at the zero and Nyquist frequencies and joint $F$-type statistics are pivotal, while those of the $t$-statistics at the harmonic seasonal frequencies depend on nuisance parameters which derive from the lag parameters characterising the linear process. Moreover, the rates on the lag truncation required for these results to hold are shown to coincide with the corresponding rates given in Chang and Park (2002); in particular, an $o\left(T^{1 / 2}\right)$ rate is shown to be sufficient.
\end{abstract}

Keywords: seasonal unit root; HEGY tests; linear process; autoregressive approximation.

JEL code: C22.

\section{Introduction}

This paper considers testing for seasonal unit roots in a univariate time-series process. In the seminal paper in the literature, Hylleberg et al. (1990) [HEGY] develop separate regression-based $t$ - and $F$ tests for unit roots at the zero, Nyquist and annual (harmonic) frequencies in the context of quarterly data. Recently, Smith, Taylor and del Bario Castro (2009) have generalised the HEGY approach to allow for an arbitrary seasonal aspect.

${ }^{*}$ This paper is forthcoming in Econometric Theory. We thank the Editor, a Co-Editor and three anonymous referees for their helpful and constructive comments on previous versions of the paper. Tomás del Barrio Castro gratefully acknowledges financial support from Ministerio de Educación y Ciencia ECO2011-23934. 
In the non-seasonal context, Said and Dickey (1984) establish that the Augmented Dickey-Fuller $[\mathrm{ADF}]$ statistics have (pivotal) Dickey-Fuller limiting null distributions in the presence of ARMA processes of unknown finite order with independently and identically distributed (iid) zero mean and constant variance innovations. This result requires that the lag length employed should increase in proportion with the sample size $T$ at rate $o\left(T^{1 / 3}\right)$. More recently, Chang and Park (2002) extend these results to allow the shocks to follow a general linear process driven by potentially heteroskedastic martingale difference innovations and show, moreover, that a weaker $o\left(T^{1 / 2}\right)$ rate condition is sufficient for the result to hold. To the best of our knowledge, however, analogous results have not been established for the HEGY-type tests. Previous contributions have either assumed that the shocks are serially uncorrelated (e.g. Smith and Taylor, 1998), or follow a finite-order autoregressive (AR) process (e.g. Burridge and Taylor, 2001, Rodrigues and Taylor, 2004a, 2004b and Smith et al., 2009) or a finite-order moving average (MA) process (del Barrio Castro and Osborn, 2011). Therefore, none allow for either the finite-order ARMA assumption of Said and Dickey (1984) or the more general linear process assumptions of Chang and Park (2002). As Taylor (2005, p.34) notes "It has been widely conjectured, but never formally proved, that an approach along the lines of that developed in regard of the ADF test by Said and Dickey (1984) will purge the effects of ARMA behavior in the shocks from the limiting null distribution of the augmented HEGY-type statistics."

It has been known since the seminal work of Box and Jenkins (1976) that seasonally observed time series can display moving average behaviour, in addition to seasonal (autoregressive) unit roots. Indeed, Box and Jenkins (1976) developed the well-known seasonal ARIMA factorisations (a prominent example of which is the so-called airline model) as a parsimonious device for modelling dependence in seasonal data. Allowing for both autoregressive and moving average behaviour is, therefore, very important when testing for unit roots in a seasonal context. ARMA behaviour in the shocks can also be a manifestation of neglected periodic autoregressive $(P A R)$ behaviour, something which has been largely overlooked in the context of the HEGY tests. A detailed discussion of the PAR class of processes is given in Ghysels and Osborn (2001, Chapter 6). As an example, the first-order stationary $P A R$ process for a series observed with period $S$, denoted $P A R(1)_{S}$, admits a stationary and invertible ARMA representation, which combines a seasonal autoregressive term with an $\mathrm{MA}(S-1)$ component.

Motivated by these considerations, in this paper we establish that the results given by Said and Dickey (1984) and Chang and Park (2002) for the ADF statistic do extend, at least in part, to the case of the augmented HEGY-type tests. Specifically, and complementing the findings of previous authors for finite order shocks of either AR or MA form, we show that, provided the order of the lag augmentation polynomial increases in proportion with the sample size at a suitable rate, then the $t$-statistics for unit roots at the zero and Nyquist frequencies, together with all the $F$-type tests employed in this context, remain pivotal in the presence of general linear processes driven by martingale difference innovations. However, this is not the case for the $t$-statistics at the harmonic seasonal frequencies, where the asymptotic null distributions depend on nuisance parameters which derive from the coefficients characterising the linear process. Moreover, we show that the rate restriction required on the order of the lag augmentation polynomial coincides with the rate given in Chang and 
Park (2002).

The remainder of the paper is organised as follows. In section 2 we outline the seasonal model, define the hypotheses of interest within that model, and briefly review the augmented HEGY-type seasonal unit root tests. The limiting null distributions of the HEGY statistics when the shocks follow a general linear process driven by martingale difference innovations is established in section 3 , along with the necessary rate conditions. Section 4 concludes. Proofs of our main results are contained in a mathematical appendix.

In the following $\stackrel{d}{\rightarrow}$ ' denotes weak convergence and ' $\stackrel{p}{\rightarrow}$ ' convergence in probability, in each case as the sample size diverges to positive infinity; ' $a:=b$ ' (' $\left.a=: b^{\prime}\right)$ indicates that $a$ is defined by $b$ ( $b$ is defined by $a) ;\lfloor\cdot\rfloor$ denotes the integer part of its argument, and $I_{p}$ denotes the $p \times p$ identity matrix. The Euclidean norm of the $k \times 1$ vector, $x$, is defined as $\|x\|:=\left(x^{\prime} x\right)^{1 / 2}$, while for the $k \times k$ matrix, $A$, we also define $\|A\|:=\max _{x}\|A x\| /\|x\|$. Finally we define i $:=\sqrt{ }(-1)$.

\section{The Seasonal Unit Root Framework}

\subsection{The Seasonal Model and Assumptions}

Consider the univariate seasonal time-series process $\left\{x_{S t+s}\right\}$ which satisfies the following data generating process (DGP)

$$
\begin{aligned}
\alpha(L) x_{S t+s} & =u_{S t+s}, s=1-S, \ldots, 0, t=1,2, \ldots, N \\
u_{S t+s} & =\psi(L) \varepsilon_{S t+s}
\end{aligned}
$$

where the positive integer $S$ denotes the number of seasons ${ }^{1}$, and $\alpha(z):=1-\sum_{j=1}^{S} \alpha_{j}^{*} z^{j}$, is an $A R(S)$ polynomial in the conventional lag operator, $L$. The error process $u_{S t+s}$ in $(2.1 \mathrm{~b})$ is taken to be a linear process with $\psi(z):=1+\sum_{j=1}^{\infty} \psi_{j} z^{j}$. Precise conditions on this process are given below. The initial conditions, $x_{1-S}, \ldots, x_{0}$, are taken to be of $o_{p}\left(T^{1 / 2}\right)$. In what follows we define $T:=S N$.

Following Chang and Park (2002), we make the following assumptions on the innovation sequence $\left\{\varepsilon_{S t+s}\right\}$ and on the coefficients of the polynomial $\psi(L)$.

Assumption A.1 Let $\left(\varepsilon_{S t+s}, \mathcal{F}_{S t+s}\right)$ be a martingale difference sequence, with filtration $\left(\mathcal{F}_{S t+s}\right)$, where $\mathcal{F}_{S t+s} \subset \mathcal{F}_{S t+s+1}$, for all $s, t$, and such that: (a) $E\left[\varepsilon_{S t+s}^{2}\right]=\sigma^{2}$, (b) $1 / N \sum_{t=1}^{N} \varepsilon_{S t+s}^{2} \stackrel{p}{\rightarrow} \sigma^{2}$ for each $s=1-S, \ldots, 0$, and (c) $E\left|\varepsilon_{S t+s}\right|^{r}<K$ with $r \geq 4$, where $K$ is some constant depending only upon $r$.

Assumption A.2 Let the polynomial $\psi(z)$ be such that: (a) $\psi(z) \neq 0$ for all $|z| \leq 1$, and (b) $\sum_{j=1}^{\infty}|j|^{\tau}\left|\psi_{j}\right|<\infty$ for some $\tau \geq 1$.

For some of the results in this paper, it will be necessary to substitute Assumption A.1 by the following somewhat stronger assumption.

\footnotetext{
${ }^{1}$ So that, for example, $S=4$ yields the case of quarterly data, $S=12$ monthly data, and $S=1$ non-seasonal data.
} 
Assumption A.1' Let $\left(\varepsilon_{S t+s}, \mathcal{F}_{S t+s}\right)$ be a martingale difference sequence, with filtration $\left(\mathcal{F}_{S t+s}\right)$, where $\mathcal{F}_{S t+s} \subset \mathcal{F}_{S t+s+1}$, for all s,t, such that: (a) $E\left(\varepsilon_{S t+s}^{2} \mid \mathcal{F}_{S t+s-1}\right)=\sigma^{2}$, and (b) $E\left|\varepsilon_{S t+s}\right|^{r}<K$ with $r \geq 4$, where $K$ is some constant depending only upon $r$.

Remark 1: Assumptions A.1, A.2 and A.1' correspond to Assumptions 1, 2 and 1', respectively, in Chang and Park (2002) - albeit condition (b) of Assumption A.1 is slightly stronger than condition (b) of Assumption 1 in Chang and Park (2002), reflecting the seasonal aspect of the data - and we therefore refer the reader to Chang and Park (2002, pp.433-434) for a detailed discussion concerning these. However, notice, in particular, that a special case of Assumption A.2 is where $u_{S t+s}$ in (2.1b) admits the causal and invertible $\operatorname{ARMA}(p, q)$ representation, $\phi(L) u_{S t+s}=\theta(L) \varepsilon_{S t+s}$, such that all the roots of $\phi(z):=1-\sum_{i=1}^{p} \phi_{p} z^{i}$ and $\theta(z):=1-\sum_{i=1}^{q} \theta_{i} z^{i}$ lie strictly outside the unit circle. Assumption A.1 is also weaker than those previously made about the innovation process, $\varepsilon_{S t+s}$, in the regression-based seasonal unit root literature, where either Assumption A.1' or the even stronger assumption that $\varepsilon_{S t+s}$ is $\operatorname{IID}\left(0, \sigma^{2}\right)$ with finite fourth moment has been adopted.

\subsection{The Seasonal Unit Root Hypotheses}

Our focus is on tests for seasonal unit roots in $\alpha(L)$ of $(2.1 \mathrm{a})$; that is, the null hypothesis of interest is

$$
H_{0}: \alpha(L)=1-L^{S}=: \Delta_{S}
$$

Under $H_{0}$ of (2.2), the DGP (2.1) of $\left\{x_{S t+s}\right\}$ is a seasonally integrated process. We may factorise the $\operatorname{AR}(S)$ polynomial $\alpha(L)$ as $\alpha(L)=\prod_{j=0}^{\lfloor S / 2\rfloor} \omega_{j}(L)$, where $\omega_{0}(L):=\left(1-\alpha_{0} L\right)$ associates the parameter $\alpha_{0}$ with the zero frequency $\omega_{0}:=0, \omega_{j}(L):=\left[1-2\left(\alpha_{j} \cos \omega_{j}-\beta_{j} \sin \omega_{j}\right) L+\left(\alpha_{j}^{2}+\beta_{j}^{2}\right) L^{2}\right]$ corresponds to the conjugate (harmonic) seasonal frequencies $\left(\omega_{j}, 2 \pi-\omega_{j}\right), \omega_{j}:=2 \pi j / S$, with associated parameters $\alpha_{j}$ and $\beta_{j}, j=1, \ldots, S^{*}$, where $S^{*}:=\lfloor(S-1) / 2\rfloor$, and, for $S$ even, $\omega_{S / 2}(L):=\left(1+\alpha_{S / 2} L\right)$, associates the parameter $\alpha_{S / 2}$ with the Nyquist frequency ${ }^{2} \omega_{S / 2}:=\pi$. Consequently $H_{0}$ of (2.2) may be commensurately partitioned as $H_{0}=\cap_{j=0}^{\lfloor S / 2\rfloor} H_{0, j}$, where

$$
H_{0, i}: \alpha_{i}=1, \quad i=0, S / 2, \text { and } H_{0, j}: \alpha_{j}=1, \beta_{j}=0, j=1, \ldots, S^{*}
$$

The hypothesis $H_{0,0}$ corresponds to a unit root at the zero frequency while $H_{0, S / 2}$ yields a unit root at the Nyquist frequency, $\omega_{S / 2}=\pi$. A pair of complex conjugate unit roots at the $j$ th harmonic seasonal frequencies is obtained under $H_{0, j}, j=1, \ldots, S^{*}$.

The alternative hypothesis $H_{1}$ is of stationarity at one or more of the zero or seasonal frequencies; that is, $H_{1}=\cup_{j=0}^{\lfloor S / 2\rfloor} H_{1, j}$, where

$$
H_{1, i}:\left|\alpha_{i}\right|<1, \quad i=0, S / 2, \text { and } H_{1, j}: \alpha_{j}^{2}+\beta_{j}^{2}<1, \quad j=1, \ldots, S^{*} .
$$

\footnotetext{
${ }^{2}$ As a point of notation, throughout the paper where reference is made to the Nyquist frequency this is understood only to apply where $S$ is even. Where $S$ is odd, elements and discussion pertaining to the Nyquist frequency should simply be deleted.
} 
Consequently, the maintained hypothesis $H_{0} \cup H_{1}$ excludes all unit roots, except for a possible single unit root at each of the zero and Nyquist frequencies and a single pair of complex conjugate unit roots at each of the harmonic seasonal frequencies. Explosive roots in $\alpha(L)$ are also excluded.

\subsection{The Augmented HEGY Tests}

In order to develop regression-based seasonal unit root tests, note first that under Assumption A.2 $\psi(z)$ is invertible, and let the (unique) inverse of $\psi(z)$ be denoted $d(z):=1-\sum_{j=1}^{\infty} d_{j} z^{j}$. Then, it follows, using the Proposition in HEGY (1990, pp.221-222) that expanding $\alpha(z) d(z)$ around the zero and seasonal frequency unit roots, $\exp ( \pm \mathrm{i} 2 \pi j / S), j=0, \ldots,\lfloor S / 2\rfloor$, that the hypotheses in (2.3) may be re-stated as $H_{0,0}: \pi_{0}=0, H_{0, S / 2}: \pi_{S / 2}=0$, and $H_{0, j}: \pi_{j}=\pi_{j}^{*}=0, j=1, \ldots, S^{*}$, in the model

$$
d^{*}(L) \Delta_{S} x_{S t+s}=\pi_{0} x_{0, S t+s}+\pi_{S / 2} x_{S / 2, S t+s}+\sum_{j=1}^{S^{*}}\left(\pi_{j} x_{j, S t+s}+\pi_{j}^{*} x_{j, S t+s}^{*}\right)+\varepsilon_{S t+s}
$$

omitting the term $\pi_{S / 2} x_{S / 2, S t+s}$ where $S$ is odd, where $d^{*}(z):=1-\sum_{j=1}^{\infty} d_{j}^{*} z^{j}$ is a causal AR polynomial, and where

$$
\begin{aligned}
x_{0, S t+s} & :=\sum_{j=0}^{S-1} x_{S t+s-j-1}, \quad x_{S / 2, S t+s}:=\sum_{j=0}^{S-1} \cos [(j+1) \pi] x_{S t+s-j-1}, \\
x_{i, S t+s} & :=\sum_{j=0}^{S-1} \cos \left[(j+1) \omega_{i}\right] x_{S t+s-j-1}, \quad x_{i, S t+s}^{*}:=-\sum_{j=0}^{S-1} \sin \left[(j+1) \omega_{i}\right] x_{S t+s-j-1}, \quad i=1, \ldots, S^{*} .
\end{aligned}
$$

As in Chang and Park (2002, p.434), we can approximate $u_{S t+s}$ from (2.1b) in $r$ th mean by the finite-order $A R$ process

$$
u_{S t+s}=d_{1} u_{S t+s-1}+\cdots+d_{k} u_{S t+s-k}+e_{S t+s}^{k}
$$

with

$$
e_{S t+s}^{k}=\varepsilon_{S t+s}+\sum_{j=k+1}^{\infty} d_{j} u_{S t+s-j} .
$$

Using the fact that under $H_{0}$ of $(2.2), \Delta_{S} x_{S t+s}=u_{S t+s}$ and $d^{*}(L)=d(L)$, substituting (2.7) into (2.5) yields the auxiliary regression equation

$$
\Delta_{S} x_{S t+s}=\pi_{0} x_{0, S t+s}+\pi_{S / 2} x_{S / 2, S t+s}+\sum_{j=1}^{S^{*}}\left(\pi_{j} x_{j, S t+s}+\pi_{j}^{*} x_{j, S t+s}^{*}\right)+\sum_{j=1}^{k} d_{j} \Delta_{S} x_{S t+s-j}+e_{S t+s}^{k}
$$

again omitting the term $\pi_{S / 2} x_{S / 2, S t+s}$ where $S$ is odd, so that (2.9) may be estimated by OLS over observations $S t+s=k+1, \ldots, T$. Consequently, as discussed for $S=4$ in HEGY and for general $S$ in Smith et al. (2009), tests for the presence (or otherwise) of a unit root at the zero and Nyquist frequencies may be obtained using conventional lower tailed regression $t$-tests, denoted $t_{0}$ and $t_{S / 2}$, for the exclusion of $x_{0, S t+s}$ and $x_{S / 2, S t+s}$, respectively, from (2.9). Similarly, the hypothesis of a pair of complex unit roots at the $j$ th harmonic seasonal frequency may be tested by the lower-tailed $t_{j}$ 
and two-tailed $t_{j}^{*}$ regression $t$-tests for the exclusion of $x_{j, S t+s}$ and $x_{j, S t+s}^{*}$, respectively, or by the (upper-tailed) regression $F$-test, denoted $F_{j}$, for the exclusion of both $x_{j, S t+s}$ and $x_{j, S t+s}^{*}$ from (2.9), $j=1, \ldots, S^{*}$. Ghysels et al. (1994) for $S=4$ and Smith et al. (2009), again for general $S$, also consider the joint frequency (upper-tail) regression $F$-tests from (2.9), namely $F_{1 \ldots\lfloor S / 2\rfloor}$ for the exclusion of $x_{S / 2, S t+s}$, together with $x_{j, S t+s}$ and $x_{j, S t+s}^{*}, j=1, \ldots, S^{*}$, and $F_{0 \ldots\lfloor S / 2\rfloor}$ for the exclusion of $x_{0, S t+s}$, $x_{S / 2, S t+s}$, and $x_{j, S t+s}$ and $x_{j, S t+s}^{*}, j=1, \ldots, S^{*}$. The former tests the null hypothesis of unit roots at all seasonal frequencies, while the latter tests the overall null, $H_{0}$.

As discussed in Chang and Park (2002, p.434), under Assumption A.2 it holds that $\sum_{j=1}^{\infty}|j|^{\tau}\left|d_{j}\right|<$ $\infty$, and consequently $\sum_{j=k+1}^{\infty}\left|d_{j}\right|=o\left(k^{-\tau}\right)$. Hence, as in Chang and Park (2002), the existence of the $r$ th moment of $u_{S t+s}$, which is implied by Assumptions A.1 and A.2, yields that

$$
E\left|e_{S t+s}^{k}-\varepsilon_{S t+s}\right|^{r} \leq E\left|u_{S t+s}\right|^{r}\left(\sum_{j=k+1}^{\infty}\left|d_{j}\right|\right)^{r}=o\left(k^{-r \tau}\right) .
$$

Consequently, the approximation error in (2.7), and hence in (2.9), becomes small as $k$ gets large. However, as in Chang and Park (2002), an assumption is still required concerning the rate permitted on the lag truncation parameter, $k$, as the sample size increases. Depending on the context, three possible assumptions can be made, as follows:

Assumption A.3 Let $k \rightarrow \infty$ and $k=o\left(T^{1 / 2}\right)$ as $T \rightarrow \infty$.

Assumption A.3' Let $k \rightarrow \infty$ and $k=o\left([T / \log T]^{1 / 2}\right)$ as $T \rightarrow \infty$.

Assumption A.3" Let $k \rightarrow \infty$ and $k=o\left(T^{1 / 3}\right)$ as $T \rightarrow \infty$.

Remark 2: As noted in Chang and Park (2002), Assumptions A.2 and A.3 are considerably weaker than the corresponding assumptions used in Said and Dickey (1984) which, in particular, rule out the possibility of a logarithmic rate on $k$. As for the non-seasonal case considered in Chang and Park (2002), we will show in the next section that the rate conditions given in either Assumptions A.3' or Assumption A.3" are sufficient to guarantee the consistency of the estimators of the coefficients, $d_{j}$, $j=1, \ldots, k$, on the lagged dependent variables in $(2.9)$. The rate imposed by the former is sufficient for consistency under homogeneous martingale difference innovations, as in Assumption A.1', while the rate imposed by the latter is sufficient for possibly heterogenous martingale difference innovations, as allowed under Assumption A.1.

\section{Asymptotic Results}

Under $H_{0}$ of $(2.2),\left\{x_{S t+s}\right\}$ of $(2.1)$ admits the so-called vector of seasons representation

$$
X_{t}=X_{t-1}+U_{t}, \quad t=1,2, \ldots, N
$$


where $X_{t}:=\left[x_{S t-(S-1)}, x_{S t-(S-2)}, \ldots, x_{S t}\right]^{\prime}, t=0, \ldots, N$, and $U_{t}:=\left[u_{S t-(S-1)}, u_{S t-(S-2)}, \ldots, u_{S t}\right]^{\prime}$, $t=1, \ldots, N$. As shown in Burridge and Taylor (2001), the error process, $U_{t}$ satisfies the vector $M A(\infty)$ representation

$$
U_{t}=\sum_{j=0}^{\infty} \boldsymbol{\Psi}_{j} E_{t-j}
$$

where $E_{t}:=\left[\varepsilon_{S t-(S-1)}, \varepsilon_{S t-(S-2)}, \ldots, \varepsilon_{S t}\right]^{\prime}$ and the $S \times S$ matrices:

$$
\boldsymbol{\Psi}_{0}:=\left[\begin{array}{cccccc}
1 & 0 & 0 & 0 & \cdots & 0 \\
\psi_{1} & 1 & 0 & 0 & \cdots & 0 \\
\psi_{2} & \psi_{1} & 1 & 0 & \cdots & 0 \\
\psi_{3} & \psi_{2} & \psi_{1} & 1 & \cdots & 0 \\
\vdots & \vdots & \vdots & \vdots & \ddots & \vdots \\
\psi_{S-1} & \psi_{S-2} & \psi_{S-3} & \psi_{S-4} & \cdots & 1
\end{array}\right]
$$

and

$$
\mathbf{\Psi}_{j}:=\left[\begin{array}{cccccc}
\psi_{j S} & \psi_{j S-1} & \psi_{j S-2} & \psi_{j S-3} & \cdots & \psi_{j S-(S-1)} \\
\psi_{j S+1} & \psi_{j S} & \psi_{j S-1} & \psi_{j S-2} & \cdots & \psi_{j S-(S-2)} \\
\psi_{j S+2} & \psi_{j S+1} & \psi_{j S} & \psi_{j S-1} & \cdots & \psi_{j S-(S-3)} \\
\psi_{j S+3} & \psi_{j S+2} & \psi_{j S+1} & \psi_{j S} & \cdots & \psi_{j S-(S-4)} \\
\vdots & \vdots & \vdots & \vdots & \ddots & \vdots \\
\psi_{j S+S-1} & \psi_{j S+S-2} & \psi_{j S+S-3} & \psi_{j S+S-4} & \cdots & \psi_{j S}
\end{array}\right], j=1,2, \ldots
$$

In Lemma 1 we now provide a multivariate invariance principle for $X_{t}$ of (3.1)-(3.2). This provides the basic building block for the asymptotic results given in this paper. In Lemma 2 we then establish the large sample properties of the focal variables $x_{j, S t+s}, j=0, \ldots,\lfloor S / 2\rfloor$, and $x_{i, S t+s}^{*}, i=1, \ldots, S^{*}$, from (2.9).

Lemma 1 Let $X_{t}$ be generated by (3.1)-(3.2). Then under Assumptions A.1 and A.2,

$$
N^{-1 / 2} X_{\lfloor r N\rfloor} \stackrel{d}{\rightarrow} \sigma \mathbf{\Psi}(1) \mathbf{W}(r):=\mathbf{B}(r), \quad r \in[0,1]
$$

where $\mathbf{W}(r)$ is a $S \times 1$ standard Brownian motion process and $\mathbf{\Psi}(1):=\sum_{j=0}^{\infty} \mathbf{\Psi}_{j}$. Notice, therefore, that $\mathbf{B}(r)$ is a $S \times 1$ vector Brownian motion process with variance matrix $\boldsymbol{\Omega}:=\sigma^{2} \Psi(1) \Psi(1)^{\prime}$. Moreover, the right member of the convergence result in (3.3) can also be written as $\frac{\sigma}{S}\left[\psi(1) C_{0}+\psi(-1) C_{S / 2}\right.$ $\left.+2 \sum_{i=1}^{S^{*}}\left(b_{i} C_{i}+a_{i} C_{i}^{*}\right)\right] \mathbf{W}(r)$, where $C_{0}:=\operatorname{Circ}[1,1,1, \ldots, 1], C_{S / 2}=\operatorname{Circ}[1,-1,1, \ldots,-1]$, and, for $\omega_{i}=2 \pi i / S, C_{i}=\operatorname{Circ}\left[\cos (0), \cos \left(\omega_{i}\right), \cos \left(2 \omega_{i}\right), \ldots, \cos \left((S-1) \omega_{i}\right)\right]$ and $C_{i}^{*}=\operatorname{Circ}[\sin (0)$, $\left.\sin \left((S-1) \omega_{i}\right), \sin \left((S-2) \omega_{i}\right), \ldots, \sin \left(\omega_{i}\right)\right], i=1, \ldots, S^{*}$, are $S \times S$ circulant matrices, and where $a_{i}:=\operatorname{Im}\left(\psi\left[\exp \left(\mathrm{i} \omega_{i}\right)\right]\right)$ and $b_{i}:=\mathcal{R} e\left(\psi\left[\exp \left(\mathrm{i} \omega_{i}\right)\right]\right), i=1, \ldots, S^{*}, \mathcal{R} e(\cdot)$ and $\mathcal{I} m(\cdot)$ denoting the real and imaginary parts of their arguments, respectively.

Remark 3: Of the circulant matrices appearing in Lemma 1, both $C_{0}$ and $C_{S / 2}$ have rank one, while $C_{j}$ and $C_{j}^{*}, j=1, \ldots, S^{*}$, are all of rank two. For further details on circulant matrices see, for example, Davis (1979), Osborn and Rodrigues (2002), and Smith et al. (2009). 
Lemma 2 Let the conditions of Lemma 1 hold. Then for $X_{j, t}:=\left[x_{j, S t-(S-1)}, x_{j, S t-(S-2)}, \ldots, x_{j, S t}\right]^{\prime}$, $j=0, \ldots,\lfloor S / 2\rfloor$, and $X_{i, t}^{*}:=\left[x_{i, S t-(S-1)}^{*}, x_{i, S t-(S-1)}^{*}, \ldots, x_{i, S t}^{*}\right]^{\prime}, i=1, \ldots, S^{*}$, we have that

$$
\begin{array}{rll}
N^{-1 / 2} X_{0,\lfloor r N\rfloor} & \stackrel{d}{\rightarrow} \sigma \psi(1) C_{0} \mathbf{W}(r) \\
N^{-1 / 2} X_{S / 2,\lfloor r N\rfloor} & \stackrel{d}{\rightarrow} \sigma \psi(-1) C_{S / 2} \mathbf{W}(r) \\
N^{-1 / 2} X_{i,\lfloor r N\rfloor} & \stackrel{d}{\rightarrow} \sigma\left(b_{i} C_{i}+a_{i} C_{i}^{*}\right) \mathbf{W}(r), \quad i=1, \ldots, S^{*} \\
N^{-1 / 2} X_{i,\lfloor r N\rfloor}^{*} & \stackrel{d}{\rightarrow} \sigma\left(b_{i} C_{i}^{*}-a_{i} C_{i}\right) \mathbf{W}(r), \quad i=1, \ldots, S^{*}
\end{array}
$$

where the vector standard Brownian motion, $\mathbf{W}(r)$, the constants $a_{i}$ and $b_{i}, i=1, \ldots, S^{*}$, and the circulant matrices, $C_{j}, j=0, \ldots,\lfloor S / 2\rfloor$, and $C_{i}^{*}, i=1, \ldots, S^{*}$, are as defined in Lemma 1.

Remark 4: It can be seen from the results in Lemma 2 that the right members of (3.4)-(3.7) are formed from linear combinations of the $S$ independent standard Brownian motions which comprise $\mathbf{W}(r)$. Recalling that $C_{0}$ and $C_{S / 2}$ both have rank one, whereas $C_{j}$ and $C_{j}^{*}$ for $j=1, \ldots, S^{*}$, all have rank two, it is seen that each element of $C_{i} \mathbf{W}(r), i=0, S / 2$, and of $C_{j} \mathbf{W}(r), C_{j}^{*} \mathbf{W}(r) j=1, \ldots, S^{*}$, is, after rescaling, a function of a scalar standard Brownian motion and of two standard Brownian motions, respectively. Moreover, since the products $C_{0} C_{S / 2}$ and $C_{i} C_{j}$ and $C_{i} C_{j}^{*}, i=0, S / 2, j=1, \ldots, S^{*}$, are all zero matrices, it is seen that these Brownian motions arising from the linear combinations which feature in the right members of (3.4) and (3.5) are independent of one another and of those which arise in the right members of (3.6) and (3.7). Moreover, by virtue of the fact that the products $C_{i} C_{j}^{*}, C_{i} C_{j}$ and $C_{i}^{*} C_{j}^{*}, i, j=1, \ldots, S^{*}, i \neq j$, are also all zero matrices, the pairs of Brownian motions featuring in (3.6) and (3.7) are also seen to be independent across $i=1, \ldots, S^{*}$.

Drawing on the results in Lemmas 1 and 2 we may now state our main result which details the large sample behaviour of the unit root statistics from (2.9) under the general linear process assumptions adopted in this paper.

Proposition 1 Let the conditions of Lemma 1 hold. Moreover, let Assumption A.3 hold in the auxiliary HEGY regression (2.9). Then the $t_{0}, t_{S / 2}$ (S even), $t_{j}$ and $t_{j}^{*}, j=1, \ldots, S^{*}$, statistics from (2.9) are such that:

$$
\begin{aligned}
& t_{i} \stackrel{d}{\rightarrow} \frac{\int_{0}^{1} W_{i} d W_{i}}{\sqrt{\int_{0}^{1} W_{i}^{2} d r}}=: \eta_{i}, \quad i=0, S / 2 \\
& t_{j} \stackrel{d}{\rightarrow} \frac{a_{j}\left[\int_{0}^{1} W_{j}^{*} d W_{j}-\int_{0}^{1} W_{j} d W_{j}^{*}\right]+b_{j}\left[\int_{0}^{1} W_{j} d W_{j}+\int_{0}^{1} W_{j}^{*} d W_{j}^{*}\right]}{\sqrt{\left(a_{j}^{2}+b_{j}^{2}\right)\left[\int_{0}^{1} W_{j}^{2} d r+\int_{0}^{1} W_{j}^{* 2} d r\right]}}, \quad j=1, \ldots, S^{*} \\
& t_{j}^{*} \stackrel{d}{\rightarrow} \frac{a_{j}\left[\int_{0}^{1} W_{j}^{*} d W_{j}^{*}+\int_{0}^{1} W_{j} d W_{j}\right]+b_{j}\left[\int_{0}^{1} W_{j} d W_{j}^{*}-\int_{0}^{1} W_{j}^{*} d W_{j}\right]}{\sqrt{\left(a_{j}^{2}+b_{j}^{2}\right)\left[\int_{0}^{1} W_{j}^{2} d r+\int_{0}^{1} W_{j}^{* 2} d r\right]}}, j=1, \ldots, S^{*}
\end{aligned}
$$

where $W_{0}, W_{S / 2}, W_{j}^{*}$ and $W_{j}, j=1, \ldots, S^{*}$, are independent standard (scalar) Brownian motions, and the constants, $a_{j}$ and $b_{j}, j=1, \ldots, S^{*}$, are as defined in Lemma 1. 
Remark 5: For the quarterly case, $S=4$, the representations given in (3.8)-(3.10) coincide with the corresponding representations given in, for example, Theorem 2.1 of Burridge and Taylor (2001). Burridge and Taylor (2001) derive their results under conditions on the innovation process $\varepsilon_{S t+s}$ which are analogous to our Assumption A.1' coupled with the much stronger assumption than our Assumption A.2 that the inverse of $\psi(z)$ is finite-ordered; that is, they assume that $u_{S t+s}$ in $(2.1 \mathrm{~b})$ follows an $A R(p)$ process with $p$ finite. Correspondingly, their results require that the lag truncation in (2.9) is a fixed number (i.e., not a function of the sample size) no smaller than $p$. Other authors have made the same or stronger assumptions than those made in Burridge and Taylor (2001). We have therefore demonstrated that the limiting distributions obtained by previous authors can be maintained under much weaker assumptions on both the serial dependence in the shocks, $u_{S t+s}$, and on the moments of the innovation process, $\varepsilon_{S t+s}$, than in this previous literature. Moreover, our results highlight the fact that the conjecture made by a number of these authors that the lag length would need to increase at rate $o\left(T^{1 / 3}\right)$ when MA behaviour is permitted in $u_{S t+s}$ to obtain this result is in fact more stringent than is necessary.

Remark 6: In the light of Remark 5 it follows immediately, as in Burridge and Taylor (2001), that the $F$-type statistics, $F_{j}, j=1, \ldots, S^{*}, F_{1 \ldots\lfloor S / 2\rfloor}$ and $F_{0 \ldots\lfloor S / 2\rfloor}$ from (2.9) have the following limiting null distributions:

$$
\begin{aligned}
& F_{j} \stackrel{d}{\rightarrow} \frac{\left[\int_{0}^{1} W_{j} d W_{j}+\int_{0}^{1} W_{j}^{*} d W_{j}^{*}\right]^{2}+\left[\int_{0}^{1} W_{j} d W_{j}^{*}-\int_{0}^{1} W_{j}^{*} d W_{j}\right]^{2}}{2\left(\int_{0}^{1} W_{j}^{2} d r+\int_{0}^{1} W_{j}^{* 2} d r\right)}:=\eta_{j}, \quad j=1, \ldots, S \\
& F_{1 \ldots\lfloor S / 2\rfloor} \stackrel{\stackrel{d}{\rightarrow}}{\rightarrow} \frac{1}{S-1}\left(\eta_{2}^{2}+2 \sum_{j=1}^{S^{*}} \eta_{j}\right), \quad F_{0 \ldots\lfloor S / 2\rfloor} \stackrel{d}{\rightarrow} \frac{1}{S}\left(\eta_{0}^{2}+\eta_{2}^{2}+2 \sum_{j=1}^{S^{*}} \eta_{j}\right)
\end{aligned}
$$

omitting $\eta_{2}^{2}$ from both expressions in (3.12) when $S$ is odd. For $S=4$, the representations given in (3.8), (3.11) and (3.12) coincide with those given for the case where $u_{S t+s}$ is serially uncorrelated in Smith and Taylor (1998, pp.279-280). The limiting null distributions of the $t_{0}, t_{S / 2}, F_{k}, k=$ $1, \ldots, S^{*}, F_{1 \ldots\lfloor S / 2\rfloor}$ and $F_{0 \ldots\lfloor S / 2\rfloor}$ statistics from (2.9) are therefore invariant to the serial correlation nuisance parameters $\left\{\psi_{j}\right\}_{j=1}^{\infty}$ which characterise the serial dependence in $u_{S t+s}$. Previously tabulated asymptotic critical values for the tests based on these statistics may therefore still be used; for example, (3.8) is the standard Dickey-Fuller distribution tabulated in Fuller (1996, Table 10.A.2, p.642).

Remark 7: Regardless of the serial dependence in $u_{S t+s}$, it is seen from the results in Proposition 1 and Remark 6 that the harmonic frequency statistics $t_{j}, t_{j}^{*}$ and $F_{j}$ are asymptotically independent across $j=1, \ldots, S^{*}$ and are asymptotically independent of the zero and Nyquist frequency statistics, $t_{0}$ and $t_{S / 2}$, respectively, under $H_{0}$ of $(2.2)$ by virtue of the mutual independence of $W_{0}, W_{j}, W_{j}^{*}$, $j=1, \ldots, S^{*}$, and $W_{S / 2}$. Moreover, $t_{0}$ is asymptotically independent of $t_{S / 2}$ and $F_{1 \ldots\lfloor S / 2\rfloor}$.

Remark 8: The proof of Proposition 1 includes the result that under Assumptions A.1, A.2 and A.3 the usual OLS residual variance estimator from $(2.9), \hat{\sigma}^{2}$ say, has the property that $\hat{\sigma}^{2} \stackrel{p}{\rightarrow} \sigma^{2}$. This result is, of course, required for using information criteria based methods, such as, for example, $A I C$ and $B I C$, to determine the lag length $k$ in $(2.9)$. 
Remark 9: Under Assumptions A.1, A.2 and A.3" it can be shown, following the same lines as in the proof of Lemma 3.4 in Chang and Park (2002), that the OLS estimator of $\Phi:=\left[d_{1}, \ldots, d_{k}\right]^{\prime}$ from (2.9) satisfies $\|\hat{\Phi}-\Phi\|=o_{p}\left(k^{-1 / 2}\right)$, for $N$ large. Moreover, under Assumptions A.1', A.2 and A.3' it can also be shown that Lemma 3.5 in Chang and Park (2002) carries over to the seasonal case and, hence, that

$$
\hat{\Phi}=\Phi+O_{p}\left(k\left[\frac{\log T}{T}\right]^{1 / 2}\right)+o_{p}\left(k^{-\tau}\right)
$$

where $\tau$ is defined in Assumption A.2. These results show which assumptions are sufficient to establish the consistency of the estimators of $d_{1}, \ldots, d_{k}$, as required to make use of sequential algorithms, such as those of Ng and Perron (1995) and Beaulieu and Miron (1993), to determine the lag length in (2.9).

Remark 10: Thus far we have considered the case where the process $\left\{x_{S t+s}\right\}$ admits no deterministic component. It is straightforward to extend the foregoing results to the case where the series contains deterministic elements. To that end, consider the following generalisation of (2.1):

$$
\begin{aligned}
x_{S t+s} & =\mu_{S t+s}+y_{S t+s}, s=1-S, \ldots, 0, \quad t=0,1, \ldots, N, \\
\alpha(L) y_{S t+s} & =u_{S t+s}, s=1-S, \ldots, 0, t=1,2, \ldots, N, \\
u_{S t+s} & =\psi(L) \varepsilon_{S t+s} .
\end{aligned}
$$

In (3.13a), $\mu_{S t+s}:=\gamma^{\prime} Z_{S t+s}$ where $Z_{S t+s}$ are purely deterministic. The right member of the auxiliary regression in (2.9) must now be correspondingly augmented by the addition of the deterministic component $\mu_{S t+s}^{*}:=\gamma^{*} Z_{S t+s}$, where $\mu_{S t+s}$ and $\mu_{S t+s}^{*}$ are linear in the mapping $\gamma \mapsto \gamma^{*}$. Smith et al. (2009) present a typology of six cases of interest for $\mu_{S t+s}$, namely: no deterministic component (as considered above); non-seasonal intercept; non-seasonal intercept and non-seasonal trend; seasonal intercepts; seasonal intercepts and non-seasonal trend, and seasonal intercepts and seasonal trends. It is important to notice, as shown in Smith et al. (2009), that the inclusion of seasonal intercepts in (2.9) renders the resulting unit root tests similar with respect to the initial conditions, $y_{1-S}, \ldots, y_{0}$. Where such deterministic components are included in (2.9), the results given in this section still hold provided the standard Brownian motions, $W_{0}, W_{1}, W_{1}^{*}, \ldots, W_{S^{*}}, W_{S^{*}}^{*}$ and (where $S$ is even) $W_{S / 2}$, are re-defined as appropriate to the deterministic scenario of interest; cf. Sections 4.1-4.5 of Smith and Taylor (1998) and Smith and Taylor (1999). As an example, if seasonal intercepts are included in (2.9) then the standard Brownian motions above are all replaced by their demeaned analogues, so that (for instance) $W_{0}$ is replaced by the process $W_{0}-\int_{0}^{1} W_{0}(s) d s$. The same can also be shown to hold for the corresponding HEGY-type tests based on local GLS de-trending as outlined in Rodrigues and Taylor (2007), again provided the standard Brownian motions are replaced by their relevant local GLS de-trended analogues; see Theorem 5.1 of Rodrgues and Taylor (2007,pp.559-560).

Remark 11: The foregoing results also extend straightforwardly to the near seasonally integrated case considered in Rodrigues and Taylor (2007,p.551). Here the polynomial $\alpha(L)$ in (2.1a) is written in local-to-unity form using $\alpha_{0}=\left(1+\frac{\nu_{0}}{T}\right), \alpha_{S / 2}=\left(1+\frac{\nu_{S / 2}}{T}\right)(S$ even$)$, and $\alpha_{k}=\left(1+\frac{\nu_{k}}{T}\right)$ and $\beta_{k}=0$, $k=1, \ldots, S^{*}$, where the non-centrality parameters, $\nu_{0}, \nu_{1}, \ldots, \nu_{\lfloor S / 2\rfloor}$, are finite constants. Under this 
framework the results given in this section continue to hold but with the standard Brownian motions, $W_{0}, W_{1}, W_{1}^{*}, \ldots, W_{S^{*}}, W_{S^{*}}^{*}$ and (where $S$ is even) $W_{S / 2}$ replaced by the corresponding OrnsteinUhlenbeck [OU] processes, as detailed in Rodrigues and Taylor (2007), so that for example $W_{0}(r)$ would be replaced by $J_{c, 0}(r):=\int_{0}^{r} \exp \left(\nu_{0}(r-\lambda)\right) d W_{0}(\lambda)$.

\section{Conclusions}

In this paper we have extended the results relating to the asymptotic null distribution for the ADF unit root tests given in Chang and Park (2002) to the case of augmented HEGY seasonal unit root tests. Specifically, we have shown that regression $t$-statistics for unit roots at the zero and Nyquist frequencies and all $F$-type statistics have pivotal limiting null distributions in the case where the shocks follow a general linear process driven by martingale difference innovations, but that this is not the case for the $t$-statistics at the harmonic seasonal frequencies whose asymptotic null distributions depend on serial correlation nuisance parameters. The (deterministic) rate at which the length of the lag augmentation polynomial used in the test regression is required to increase for these results to hold was also explored and shown to coincide with the rate derived for the non-seasonal ADF statistic by Chang and Park (2002).

The focus of this paper has been to establish the theoretical validity of the use of lag augmentation (of deterministic order) in the HEGY seasonal unit root tests, paralleling the contribution of Chang and Park (2002) for ADF tests. Empirical practice, however, takes matters a stage further and employs data-dependent methods to select the lag augmentation polynomial. In the accompanying working paper, del Barrio Castro, Osborn and Taylor (2011), we use Monte Carlo methods to explore and compare the performance of a variety of such procedures: the sequential test procedures employed by Hall (1994) and Ng and Perron (1995), seasonal variants of these as suggested by Rodrigues and Taylor (2004a) and Beaulieu and Miron (1993), and methods based on information criteria including $A I C, B I C$ and a seasonal generalisation of the modified information criteria $[M A I C, M B I C]$ of $\mathrm{Ng}$ and Perron (2001). Our findings can be summarised as follows. In general, the procedure of Beaulieu and Miron (1993) applied using a 10\% significance level on the lag specification tests, was shown to perform well, when taking both size and power considerations into account. This method avoided the severe size distortions seen in the presence of ARMA shocks by many of the procedures and at the same time was generally competitive on power with the other procedures. Deleting intermediate lags, in the spirit of the seasonal factorisations of Box and Jenkins (1976), was shown to be advantageous in terms of power, but it was also shown that such deletion could lead to increased size distortions, especially in the presence of MA disturbances. The results showed that information criteria approaches could have potentially very poor size (for example, $A I C / B I C$ with MAs) or poor power $(M A I C / M B I C$ for disturbance processes with negative seasonal AR coefficients). However, of all the procedures considered, the most reliable size properties were demonstrated by the $M A I C$ method of $\mathrm{Ng}$ and Perron (2001) even in cases where a near-cancellation occurs across AR unit roots with corresponding roots in the MA disturbance process. 


\section{References}

Beaulieu, J.J., and Miron, J.A., (1993). Seasonal unit roots in aggregate U.S. data. Journal of Econometrics 55, 305-328.

Berk, K.N., (1974) Consistent autoregressive spectral estimates. The Annals of Statistics 2, 389-502.

Boswijk, H.P., and Franses, P.H., (1996). Unit roots in periodic autoregressions. Journal of Time Series Analysis 17, 221-245.

Box, G.E.P., and Jenkins, G.M., (1976). Time Series Analysis: Forecasting and Control (revised edition). San Francisco: Holden-Day.

Burridge, P., and Taylor, A. M. R., (2001). On the properties of regression-based tests for seasonal unit roots in the presence of higher-order serial correlation. Journal of Business and Economic Statistics 3, 374-379.

Chang, Y., and Park, J.Y., (2002). On the asymptotics of ADF tests for unit roots. Econometric Reviews 21, 431-447.

Davis, P.J., (1979). Circulant Matrices. Wiley-Interscience: New York.

Davidson, J., (1994). Stochastic Limit Theory. Oxford University Press: Oxford.

del Barrio Castro, T., and Osborn D.R., (2011). HEGY tests in the presence of moving averages. Oxford Bulletin of Economic and Statistics, forthcoming.

del Barrio Castro, T., Osborn D.R., and Taylor, A.M.R., (2011). On Augmented HEGY Tests for Seasonal Unit Roots. Granger Centre for Time Series Econometrics, Working Paper 11/02.

Fuller, W.A., (1996). Introduction to Statistical Time Series, Second Edition. Wiley: New York.

Ghysels, E., Lee, H. S., and Noh, J., (1994). Testing for unit roots in seasonal time series: some theoretical extensions and a Monte Carlo investigation. Journal of Econometrics 62, 415- 442,

Ghysels, E. and Osborn, D.R., (2001). The Econometric Analysis of Seasonal Time Series. Cambridge University Press: Cambridge.

Gray, R. M., (2006). Toeplitz and Circulant Matrices, A Review. Foundation and Trends(R) in Communications and Information Theory: Now Publishers Inc.

Hall, A.R., (1994). Testing for a unit root in time series with pretest data-based model selection. Journal of Business and Economic Statistics 12, 461-70.

Hamilton J., (1994). Time Series Analysis. Princeton University Press: Princeton.

Hylleberg, S., Engle, R. F., Granger, C. W. J., and Yoo, B. S., (1990). Seasonal integration and cointegration. Journal of Econometrics 44, 215-238. 
Ng, S., and Perron, P., (1995). Unit root tests in ARMA models with data dependent methods for selection of the truncation lag. Journal of the American Statistical Association 90, 268-281.

Ng, S., and Perron, P., (2001). Lag length selection and the construction of unit root tests with good size and power. Econometrica 69, 1519-1554.

Osborn, D. R., and Rodrigues, P. M. M., (2002). Asymptotic distributions of seasonal unit root tests: a unifying approach. Econometric Reviews 21, 221-241.

Phillips, P.C.B., and Durlauf, S.N., (1986). Multiple time series regression with integrated processes. Review of Economic Studies 53, 473-95.

Rodrigues, P. M. M., and Taylor A. M. R., (2004a). Alternative estimators and unit root tests for seasonal autoregressive processes. Journal of Econometrics 120, 35-73.

Rodrigues, P. M. M., and Taylor A. M. R., (2004b). Asymptotic distributions for regression-based seasonal unit root test statistics in a near-integrated model. Econometric Theory 20, 645-670.

Rodrigues, P.M.M., and Taylor, A. M. R., (2007). Efficient Tests of the Seasonal Unit Root Hypothesis. Journal of Econometrics 141, 548-573.

Said, S.E., and Dickey, D. A., (1984). Testing for unit roots in autoregressive-moving average models of unknown order. Biometrika 71, 599-607.

Smith R.J., and Taylor, A.M.R., (1998). Additional critical values and asymptotic representations for seasonal unit root tests. Journal of Econometrics 85, 269-288.

Smith, R.J., and Taylor, A.M.R., (1999). Likelihood ratio tests for seasonal unit roots. Journal of Time Series Analysis 20, 453-476.

Smith R.J., Taylor, A.M.R., and del Barrio Castro, T., (2009). Regression-based seasonal unit root tests. Econometric Theory 25, 527-560.

Taylor, A. M. R., (2005). Variance ratio tests of the seasonal unit root hypothesis. Journal of Econometrics 124, 33-54. 


\section{A Appendix}

For the purposes of the Appendix, and without loss of generality, we simplify our exposition by setting the initial vector $X_{0}:=\left(x_{1-S}, \ldots, x_{0}\right)^{\prime}=\mathbf{0}$ throughout.

Proof of Lemma 1: The conditions placed on $\left\{E_{t}\right\}$ through Assumption A.1 imply that it satisfies a multivariate invariance principle, see for example Phillips and Durlauf (1996) or Davidson (1994, pp.454-455), such that

$$
N^{-1 / 2} \sum_{j=1}^{\lfloor r N\rfloor} E_{j} \stackrel{d}{\rightarrow} \sigma \mathbf{W}(r)
$$

where $\mathbf{W}(r)$ is a $S \times 1$ vector standard Brownian motion. Next observe from (3.1) and (3.2), that

$$
\begin{aligned}
N^{-1 / 2} X_{\lfloor r N\rfloor} & =N^{-1 / 2} \sum_{j=1}^{\lfloor r N\rfloor} U_{j} \\
& =\Psi(1) N^{-1 / 2} \sum_{j=1}^{\lfloor r N\rfloor} E_{j}+o_{p}(1)
\end{aligned}
$$

where the approximation in the second line follows from the same argument as in Boswijk and Franses (1996, p.238). Using (A.14) and the continuous mapping theorem [CMT] the result in (3.3) then follows immediately.

To establish the second part of the lemma, observe first that $I_{S}=\frac{1}{S} C_{0}+\frac{1}{S} C_{S / 2}+\frac{2}{S} \sum_{j=1}^{S^{*}} C_{j}$. Moreover, noting that $\boldsymbol{\Psi}(1)$ is also a circulant matrix, then by the properties of products of circulant matrices it can be shown that $C_{0} \boldsymbol{\Psi}(1)=\psi(1) C_{0}, C_{S / 2} \boldsymbol{\Psi}(1)=\psi(-1) C_{S / 2}, C_{j} \boldsymbol{\Psi}(1)=b_{j} C_{j}+a_{j} C_{j}^{*}$ and $C_{j}^{*} \boldsymbol{\Psi}(1)=-a_{j} C_{j}+b_{j} C_{j}^{*}$ for $j=1, \ldots S^{*}$; see, inter alia, Theorem 3.2.4 of Davis (1979), Theorem 3.1 of Gray (2006) and Smith et al. (2009) for further details. The stated result then follows immediately.

Proof of Lemma 2: Noting that $X_{j, t}=C_{j} X_{t}, j=0, \ldots,\lfloor S / 2\rfloor$, and that $X_{i, t}^{*}=C_{i}^{*} X_{t}, i=1, \ldots, S^{*}$, the stated results follow immediately from Lemma 1, using the following identities: $C_{0} C_{0}=S C_{0}$, $C_{S / 2} C_{S / 2}=S C_{S / 2}, C_{j} C_{j}=\frac{S}{2} C_{j}, C_{j} C_{j}^{*}=\frac{S}{2} C_{j}^{*}$ and $C_{j}^{*} C_{j}^{*}=\frac{S}{2} C_{j}, j=1, \ldots, S^{*}$, also recalling from Remark 4 that the remaining matrix products between $C_{0}, C_{S / 2}, C_{j}$ and $C_{j}^{*}, j=1, \ldots, S^{*}$ are all zero matrices, and noting that multiplication between circulant matrices is commutative.

For later reference, noting that $C_{0}=\mathbf{v}_{0} \mathbf{v}_{0}{ }^{\prime}$, where $\mathbf{v}_{0}{ }^{\prime}=[1,1,1, \ldots, 1], C_{S / 2}=\mathbf{v}_{S / 2} \mathbf{v}_{S / 2}{ }^{\prime}$, where $\mathbf{v}_{S / 2}{ }^{\prime}=[-1,1,-1, \ldots, 1]$, and that $C_{j}=\mathbf{v}_{j} \mathbf{v}_{j}^{\prime}$ and $C_{j}^{*}=\mathbf{v}_{j} \mathbf{v}_{j}^{* \prime}$, where

$$
\mathbf{v}_{j}^{\prime}=\left[\begin{array}{cccc}
\cos \left(\omega_{j}[1-S]\right) & \cos \left(\omega_{j}[2-S]\right) & \cdots & \cos (0) \\
\sin \left(\omega_{j}[1-S]\right) & \sin \left(\omega_{j}[2-S]\right) & \cdots & \sin (0)
\end{array}\right]
$$

and

$$
\mathbf{v}_{j}^{* \prime}=\left[\begin{array}{cccc}
-\sin \left(\omega_{j}[1-S]\right) & -\sin \left(\omega_{j}[2-S]\right) & \cdots & -\sin (0) \\
\cos \left(\omega_{j}[1-S]\right) & \cos \left(\omega_{j}[2-S]\right) & \cdots & \cos (0)
\end{array}\right]
$$


it is straightforwardly seen that

$$
\begin{aligned}
N^{-1 / 2} x_{0, S\lfloor r N\rfloor+s} & =\frac{\psi(1)}{\sqrt{N}}\left(\sum_{h=1}^{S}\left[\sum_{j=1}^{\lfloor r N\rfloor} \varepsilon_{S j-(S-h)}\right]\right)+o_{p}(1) \\
N^{-1 / 2} x_{S / 2, S\lfloor r N\rfloor+s} & =\frac{\psi(-1)(-1)^{s}}{\sqrt{N}}\left(\sum_{h=1}^{S}\left[(-1)^{h} \sum_{j=1}^{\lfloor r N\rfloor} \varepsilon_{S j-(S-h)}\right]\right)+o_{p}(1)
\end{aligned}
$$

and that for $i=1, \ldots, S^{*}$,

$$
\begin{aligned}
N^{-1 / 2} x_{i, S\lfloor r N\rfloor+s}= & \frac{b_{i}}{\sqrt{N}}\left[\cos \left(\omega_{i}[s]\right)\left(\sum_{h=1}^{S} \cos \left(\omega_{i}[h-S]\right)\left[\sum_{j=1}^{\lfloor r N\rfloor} \varepsilon_{S j-(s-h)}\right]\right)\right. \\
& \left.+\sin \left(\omega_{i}[s]\right)\left(\sum_{h=1}^{S} \sin \left(\omega_{i}[h-S]\right)\left[\sum_{j=1}^{\lfloor r N\rfloor} \varepsilon_{S j-(s-h)}\right]\right)\right] \\
& +\frac{a_{i}}{\sqrt{N}}\left[\sin \left(\omega_{i}[s]\right)\left(\sum_{h=1}^{S} \cos \left(\omega_{i}[h-S]\right)\left[\sum_{j=1}^{\lfloor r N\rfloor} \varepsilon_{S j-(s-h)}\right]\right)\right. \\
& \left.-\cos \left(\omega_{i}[s]\right)\left(\sum_{h=1}^{S} \sin \left(\omega_{i}[h-S]\right)\left[\sum_{j=1}^{\lfloor r N\rfloor} \varepsilon_{S j-(s-h)}\right]\right)\right]+o_{p}(1)
\end{aligned}
$$

and

$$
\begin{aligned}
N^{-1 / 2} x_{i, S\lfloor r N\rfloor+s}^{*}= & \frac{b_{i}}{\sqrt{N}}\left[\sin \left(\omega_{i}[s]\right)\left(\sum_{h=1}^{S} \cos \left(\omega_{i}[h-S]\right)\left[\sum_{j=1}^{\lfloor r N\rfloor} \varepsilon_{S j-(s-h)}\right]\right)\right. \\
& \left.-\cos \left(\omega_{i}[s]\right)\left(\sum_{h=1}^{S} \sin \left(\omega_{i}[h-S]\right)\left[\sum_{j=1}^{\lfloor r N\rfloor} \varepsilon_{S j-(s-h)}\right]\right)\right] \\
& -\frac{a_{i}}{\sqrt{N}}\left[\cos \left(\omega_{i}[s]\right)\left(\sum_{h=1}^{S} \cos \left(\omega_{i}[h-S]\right)\left[\sum_{j=1}^{\lfloor r N\rfloor} \varepsilon_{S j-(s-h)}\right]\right)\right. \\
& \left.+\sin \left(\omega_{i}[s]\right)\left(\sum_{h=1}^{S} \sin \left(\omega_{i}[h-S]\right)\left[\sum_{j=1}^{\lfloor r N\rfloor} \varepsilon_{S j-(s-h)}\right]\right)\right]+o_{p}(1) .
\end{aligned}
$$

Proof of Proposition 1: First re-write (2.9) in vector form, viz,

$$
\mathbf{y}=\left(\mathbf{Y}, \mathbf{Z}_{k}\right) \boldsymbol{\beta}_{0}+\mathbf{u}
$$

where $\mathbf{y}$ is a $T \times 1$ vector with generic element $\Delta_{S} x_{S t+s} ; \mathbf{Y}:=\left[\mathbf{y}_{0}\left|\mathbf{y}_{1}\right| \mathbf{y}_{1}^{*}\left|\mathbf{y}_{2}\right| \mathbf{y}_{2}^{*}\left|\ldots \mathbf{y}_{S^{*}}\right| \mathbf{y}_{S^{*}}^{*} \mid \mathbf{y}_{S / 2}\right]$ is a $T \times S$ matrix where $\mathbf{y}_{i}, i=0, \ldots,\lfloor S / 2\rfloor$ are $T \times 1$ vectors with generic element $x_{i, S t+s}$, and $\mathbf{y}_{i}^{*}, i=1, \ldots, S^{*}$ are $T \times 1$ vectors with generic element $x_{i, S t+s}^{*} ; \mathbf{Z}_{k}:=\left[\mathbf{z}_{1}\left|\mathbf{z}_{2}\right| \cdots \mid \mathbf{z}_{k}\right]$ is a $T \times k$ matrix with $\mathbf{z}_{j}$ being $T \times 1$ vectors with generic element $\Delta_{S} x_{S t+s-j}$ for $j=1, \ldots, k ; \boldsymbol{\beta}_{0}=\left[\Pi^{\prime} \mid \Phi^{\prime}\right]^{\prime}$ $:=\left[\pi_{0}, \pi_{1}, \pi_{1}^{*}, \pi_{2}, \pi_{2}^{*}, \ldots, \pi_{S^{*}}, \pi_{S^{*}}^{*}, \pi_{S / 2}, d_{1}, \ldots, d_{k}\right]^{\prime}$; finally $\mathbf{u}$ is a $T \times 1$ vector with generic element $e_{S t+s}^{k}$. Commensurate with the partitions of $\boldsymbol{\beta}_{0}$, define the $(S+k) \times(S+k)$ scaling matrix, $M:=$ 
$\operatorname{diag}\left[T, \ldots, T, T^{1 / 2}, \ldots, T^{1 / 2}\right]$. It is then straightforwardly seen that the OLS estimator, $\hat{\boldsymbol{\beta}}_{0}$ say, from (2.9) is such that

$$
M\left(\hat{\boldsymbol{\beta}}_{0}-\boldsymbol{\beta}_{0}\right)=\left[\begin{array}{cc}
T^{-2} \mathbf{Y}^{\prime} \mathbf{Y} & T^{-3 / 2} \mathbf{Y}^{\prime} \mathbf{Z}_{k} \\
T^{-3 / 2} \mathbf{Z}_{k}^{\prime} \mathbf{Y} & T^{-1} \mathbf{Z}_{k}^{\prime} \mathbf{Z}_{k}
\end{array}\right]^{-1} \times\left[\begin{array}{c}
T^{-1} \mathbf{Y}^{\prime} \mathbf{u} \\
T^{-1 / 2} \mathbf{Z}_{k}^{\prime} \mathbf{u}
\end{array}\right] .
$$

Due to the unit root non-stationary of the elements of $\mathbf{Y}$ and the stationarity of the elements of $\mathbf{Z}_{k}$, it follows immediately that $T^{-3 / 2} \mathbf{Y}^{\prime} \mathbf{Z}_{k} \stackrel{p}{\rightarrow} \mathbf{0}$, so that the inverse matrix in (A.19) is asymptotically block diagonal. As a consequence, the scaled estimators $T(\hat{\Pi}-\Pi)$ and $T^{1 / 2}(\hat{\Phi}-\Phi)$ are asymptotically orthogonal. Moreover, it is straightforward to show that $T^{-2} \mathbf{Y}^{\prime} \mathbf{Y}$ weakly converges to a $S \times S$ diagonal matrix. We may therefore consider the large sample behaviour of the OLS estimators of $\pi_{j}$, $j=0, \ldots,\lfloor S / 2\rfloor$, and $\pi_{i}^{*}, i=1, \ldots, S^{*}$, separately.

Consequently, defining $\mathbf{Q}:=I_{T}-\mathbf{Z}_{k}\left(\mathbf{Z}_{k}^{\prime} \mathbf{Z}_{k}\right)^{-1} \mathbf{Z}_{k}^{\prime}$, we can write the so-called normalized bias statistics as follows:

$$
\begin{aligned}
T \widehat{\pi}_{j} & =\frac{T^{-1} \mathbf{y}_{j}^{\prime} \mathbf{Q u}}{T^{-2} \mathbf{y}_{j}^{\prime} \mathbf{Q} \mathbf{y}_{j}}+o_{p}(1) \\
& =\frac{T^{-1}\left(\mathbf{y}_{j}^{\prime} \mathbf{u}-\mathbf{y}_{j}^{\prime} \mathbf{Z}_{k}\left(\mathbf{Z}_{k}^{\prime} \mathbf{Z}_{k}\right)^{-1} \mathbf{Z}_{k}^{\prime} \mathbf{u}\right)}{T^{-2}\left(\mathbf{y}_{j}^{\prime} \mathbf{y}_{j}-\mathbf{y}_{j}^{\prime} \mathbf{Z}_{k}\left(\mathbf{Z}_{k}^{\prime} \mathbf{Z}_{k}\right)^{-1} \mathbf{Z}_{k}^{\prime} \mathbf{y}_{j}\right)}+o_{p}(1), \quad j=0, \ldots,\lfloor S / 2\rfloor
\end{aligned}
$$

and

$$
\begin{aligned}
T \widehat{\pi}_{i}^{*} & =\frac{T^{-1} \mathbf{y}_{i}^{* \prime} \mathbf{Q u}}{T^{-2} \mathbf{y}_{i}^{* \prime} \mathbf{Q} \mathbf{y}_{i}^{*}}+o_{p}(1) \\
& =\frac{T^{-1}\left(\mathbf{y}_{i}^{* \prime} \mathbf{u}-\mathbf{y}_{i}^{* \prime} \mathbf{Z}_{k}\left(\mathbf{Z}_{k}^{\prime} \mathbf{Z}_{k}\right)^{-1} \mathbf{Z}_{k}^{\prime} \mathbf{u}\right)}{T^{-2}\left(\mathbf{y}_{i}^{* \prime} \mathbf{y}_{i}^{*}-\mathbf{y}_{i}^{* \prime} \mathbf{Z}_{k}\left(\mathbf{Z}_{k}^{\prime} \mathbf{Z}_{k}\right)^{-1} \mathbf{Z}_{k}^{\prime} \mathbf{y}_{j}^{*}\right)}+o_{p}(1), j=1, \ldots, S^{*} .
\end{aligned}
$$

The following lemma will allow us to simplify the expressions given above.

Lemma 1 Under the conditions of Proposition 1 the following results hold: (i) $\left\|\mathbf{Z}_{k}^{\prime} \mathbf{Z}_{k}^{-1}\right\|=O_{p}\left(T^{-1}\right.$ ); (ii) $\left\|\mathbf{Z}_{k}^{\prime} \mathbf{u}\right\|=o_{p}\left(T k^{-1 / 2}\right)$, and (iii) $\left\|\mathbf{Z}_{k}^{\prime} \mathbf{y}_{j}\right\|=O_{p}\left(T k^{1 / 2}\right), j=0, \ldots,\lfloor S / 2\rfloor$ and $\left\|\mathbf{Z}_{k}^{\prime} \mathbf{y}_{h}^{*}\right\|=O_{p}\left(T k^{1 / 2}\right)$, $h=1, \ldots, S^{*}$.

Proof: Parts (i) and (ii) follow immediately from parts (a) and (c) respectively of Lemma 3.2 of Chang and Park (2002) simply by replacing the standard first differences which appear there by the seasonal differences, $\Delta_{S} x_{S t+s}$. In order to establish the validity of part (iii), first use the fact that for $j=0, \ldots,\lfloor S / 2\rfloor,\left\|\mathbf{Z}_{k}^{\prime} \mathbf{y}_{j}\right\|^{2}=\sum_{i=1}^{k}\left(\mathbf{z}_{i}^{\prime} \mathbf{y}_{j}\right)^{2}$, where $\mathbf{z}_{i}^{\prime} \mathbf{y}_{j}=\sum_{t=1}^{N} \sum_{s=1-S}^{0} \Delta_{S} x_{S t+s-i} x_{j, S t+s}, i=1, \ldots, k$, and, for $h=1, \ldots, S^{*},\left\|\mathbf{Z}_{k}^{\prime} \mathbf{y}_{h}^{*}\right\|^{2}=\sum_{i=1}^{k}\left(\mathbf{z}_{i}^{\prime} \mathbf{y}_{h}^{*}\right)^{2}$, where $\mathbf{z}_{i}^{\prime} \mathbf{y}_{h}^{*}=\sum_{t=1}^{N} \sum_{s=1-S}^{0} \Delta_{S} x_{S t+s-i} x_{h, S t+s}^{*} i=$ $1, \ldots, k$. Next observe that the following equalities hold for each $i=1, \ldots, k$ :

$$
\begin{aligned}
\sum_{t=1}^{N} \sum_{s=1-S}^{0} x_{0, S t+s} \Delta_{S} x_{S t+s-i} & =\sum_{t=1}^{N} \sum_{s=1-S}^{0}\left(\sum_{j=1}^{(S t+s)-1} \Delta_{S} x_{S t+s-j}\right) \Delta_{S} x_{S t+s-i} \\
\sum_{t=1}^{N} \sum_{s=1-S}^{0} x_{S / 2, S t+s} \Delta_{S} x_{S t+s-i} & =\sum_{t=1}^{N} \sum_{s=1-S}^{0}\left(\sum_{j=1}^{(S t+s)-1}(-1)^{j} \Delta_{S} x_{S t+s-j}\right) \Delta_{S} x_{S t+s-i}
\end{aligned}
$$


and, moreover, that for each $h=1, \ldots, S^{*}$, the following equalities also hold for each $i=1, \ldots, k$ :

$$
\begin{aligned}
\sum_{t=1}^{N} \sum_{s=1-S}^{0} x_{h, S t+s} \Delta_{S} x_{S t+s-i} & =\sum_{t=1}^{N} \sum_{s=1-S}^{0}\left(\sum_{j=1}^{(S t+s)-1} \cos \left(j \omega_{h}\right) \Delta_{S} x_{S t+s-j}\right) \Delta_{S} x_{S t+s-i} \\
\sum_{t=1}^{N} \sum_{s=1-S}^{0} x_{h, S t+s}^{*} \Delta_{S} x_{S t+s-i} & =\sum_{t=1}^{N} \sum_{s=1-S}^{0}\left(\sum_{j=1}^{(S t+s)-1}-\sin \left(j \omega_{h}\right) \Delta_{S} x_{S t+s-j}\right) \Delta_{S} x_{S t+s-i} .
\end{aligned}
$$

The stated result is then established using (A.22)-(A.25) and following along the lines of the proof of part (b) of Lemma 3.2 in Chang and Park (2002, pp.443-444).

Applying the results in Lemma A.1 to (A.20) and (A.21), we then have that

$$
\begin{aligned}
T \widehat{\pi}_{j} & =\frac{T^{-1} \mathbf{y}_{j}^{\prime} \mathbf{u}}{T^{-2} \mathbf{y}_{j}^{\prime} \mathbf{y}_{j}}+o_{p}(1) \\
& =\frac{T^{-1} \sum_{t=1}^{N} \sum_{s=1-S}^{0} x_{j, S t+s} e_{S t+s}^{k}}{T^{-2} \sum_{t=1}^{N} \sum_{s=1-S}^{0} x_{j, S t+s}^{2}}+o_{p}(1), \quad j=0, \ldots,\lfloor S / 2\rfloor
\end{aligned}
$$

and

$$
\begin{aligned}
T \widehat{\pi}_{h}^{*} & =\frac{T^{-1} \mathbf{y}_{h}^{* \prime} \mathbf{u}}{T^{-2} \mathbf{y}_{h}^{* \prime} \mathbf{y}_{h}^{*}}+o_{p}(1) \\
& =\frac{T^{-1} \sum_{t=1}^{N} \sum_{s=1-S}^{0} x_{h, S t+s}^{*} e_{S t+s}^{k}}{T^{-2} \sum_{t=1}^{N} \sum_{s=1-S}^{0} x_{h, S t+s}^{* 2}}+o_{p}(1), \quad h=1, \ldots, S^{*}
\end{aligned}
$$

For each of $j=0, \ldots,\lfloor S / 2\rfloor$ in (A.26) and $h=1, \ldots, S^{*}$ in (A.27), re-write the numerators of (A.26) and (A.27), respectively, as

$$
\begin{aligned}
T^{-1} \sum_{t=1}^{N} \sum_{s=1-S}^{0} x_{j, S t+s} e_{S t+s}^{k} & =T^{-1} \sum_{t=1}^{N} \sum_{s=1-S}^{0} x_{j, S t+s} \varepsilon_{S t+s}+T^{-1} \sum_{t=1}^{N} \sum_{s=1-S}^{0} x_{j, S t+s}\left(e_{S t+s}^{k}-\varepsilon_{S t+s}\right) \\
T^{-1} \sum_{t=1}^{N} \sum_{s=1-S}^{0} x_{h, S t+s}^{*} e_{S t+s}^{k} & =T^{-1} \sum_{t=1}^{N} \sum_{s=1-S}^{0} x_{h, S t+s}^{*} \varepsilon_{S t+s}+T^{-1} \sum_{t=1}^{N} \sum_{s=1-S}^{0} x_{h, S t+s}^{*}\left(e_{S t+s}^{k}-\varepsilon_{S t+s}\right) .
\end{aligned}
$$

Substituting into (A.26) and (A.27), respectively, we therefore obtain that for, $j=0, \ldots,\lfloor S / 2\rfloor$,

$$
T \widehat{\pi}_{j}=\frac{T^{-1} \sum_{t=1}^{N} \sum_{s=1-S}^{0} x_{j, S t+s} \varepsilon_{S t+s}+T^{-1} \sum_{t=1}^{N} \sum_{s=1-S}^{0} x_{j, S t+s}\left(e_{S t+s}^{k}-\varepsilon_{S t+s}\right)}{T^{-2} \sum_{t=1}^{N} \sum_{s=1-S}^{0} x_{j, S t+s}^{2}}+o_{p}(1)
$$

and that, for $h=1, \ldots, S^{*}$,

$$
T \widehat{\pi}_{h}^{*}=\frac{T^{-1} \sum_{t=1}^{N} \sum_{s=1-S}^{0} x_{h, S t+s}^{*} \varepsilon_{S t+s}+T^{-1} \sum_{t=1}^{N} \sum_{s=1-S}^{0} x_{h, S t+s}^{*}\left(e_{S t+s}^{k}-\varepsilon_{S t+s}\right)}{T^{-2} \sum_{t=1}^{N} \sum_{s=1-S}^{0} x_{h, S t+s}^{* 2}}+o_{p}(1) .
$$


Consider first the denominators of (A.28) and (A.29). Using the results $\boldsymbol{\Psi}(1)^{\prime} C_{0} \boldsymbol{\Psi}(1)=\psi(1)^{2} C_{0}$, $\boldsymbol{\Psi}(1)^{\prime} C_{S / 2} \boldsymbol{\Psi}(1)=\psi(-1)^{2} C_{S / 2}$ and $\boldsymbol{\Psi}(1)^{\prime} C_{j} \boldsymbol{\Psi}(1)=\left(a_{j}^{2}+b_{j}^{2}\right) C_{j}$, for $j=1, \ldots, S^{*}$ from the multivariate invariance principle in (3.3) and the CMT we obtain that

$$
\begin{aligned}
T^{-2} \sum_{t=1}^{N} \sum_{s=1-S}^{0} x_{j, S t+s}^{2} & =T^{-2} \sum_{t=1}^{N} S\left(X_{t-1}^{\prime} C_{j} X_{t-1}\right)+o_{p}(1) \quad j=0, S / 2 \\
& \stackrel{d}{\rightarrow} \frac{\sigma^{2}}{S} \int_{0}^{1} \mathbf{W}(r)^{\prime} \mathbf{\Psi}(1)^{\prime} C_{j} \mathbf{\Psi}(1) \mathbf{W}(r) d r \\
& =\left\{\begin{array}{rr}
\sigma^{2} \psi(1)^{2} \int_{0}^{1} \mathbf{W}^{*}(r)^{\prime} C_{0} \mathbf{W}^{*}(r) d r & j=0 \\
\sigma^{2} \psi(-1)^{2} \int_{0}^{1} \mathbf{W}^{*}(r)^{\prime} C_{S / 2} \mathbf{W}^{*}(r) d r & j=S / 2
\end{array}\right.
\end{aligned}
$$

where $\mathbf{W}^{*}(r):=\frac{1}{\sqrt{S}} \mathbf{W}(r)$ and

$$
\begin{aligned}
T^{-2} \sum_{t=1}^{N} \sum_{s=1-S}^{0} x_{j, S t+s}^{2} & =T^{-2} \sum_{t=1}^{N}\left(\frac{S}{2}\right)\left(X_{t-1}^{\prime} C_{j} X_{t-1}\right)+o_{p}(1) \quad j=1, \ldots, S^{*} \\
& \stackrel{d}{\rightarrow} \frac{\sigma^{2}}{S^{2}}\left(\frac{S}{2}\right) \int_{0}^{1} \mathbf{W}(r)^{\prime} \mathbf{\Psi}(1)^{\prime} C_{j} \mathbf{\Psi}(1) \mathbf{W}(r) d r \\
& =\frac{\sigma^{2}\left(a_{j}^{2}+b_{j}^{2}\right)}{4} \int_{0}^{1} \mathbf{W}^{\dagger}(r)^{\prime} C_{j} \mathbf{W}^{\dagger}(r) d r
\end{aligned}
$$

where $\mathbf{W}^{\dagger}(r):=\frac{1}{\sqrt{S / 2}} \mathbf{W}(r)$.

Consider next the numerators of (A.28) and (A.29). In each case, for the first term it is straightforward to show that

$$
\begin{aligned}
T^{-1} \sum_{t=1}^{N} \sum_{s=1-S}^{0} x_{j, S t+s} \varepsilon_{S t+s} & =T^{-1} \sum_{t=1}^{N} X_{t-1}^{\prime} C_{j} E_{t}+o_{p}(1), \quad j=0, \ldots,\lfloor S / 2\rfloor \\
T^{-1} \sum_{t=1}^{N} \sum_{s=1-S}^{0} x_{h, S t+s}^{*} \varepsilon_{S t+s} & =T^{-1} \sum_{t=1}^{N} X_{t-1}^{\prime} C_{h}^{*} E_{t}+o_{p}(1) \quad h=1, \ldots, S^{*} .
\end{aligned}
$$

Again using (3.3), applications of the CMT and the identities: $\boldsymbol{\Psi}(1)^{\prime} C_{0}=\psi(1) C_{0}, \Psi(1)^{\prime} C_{S / 2}=$ $\psi(-1) C_{S / 2}$, and, for $j=1, \ldots, S^{*}, \Psi(1)^{\prime} C_{j}=b_{j} C_{j}-a_{j} C_{j}^{*}$ and $\boldsymbol{\Psi}(1)^{\prime} C_{j}^{*}=a_{j} C_{j}+b_{j} C_{j}^{*}$, the following results then obtain

$$
\begin{aligned}
T^{-1} \sum_{t=1}^{N} \sum_{s=1-S}^{0} x_{0, S t+s} \varepsilon_{S t+s} & \stackrel{d}{\rightarrow} \frac{\sigma^{2}}{S} \int_{0}^{1} \mathbf{W}(r)^{\prime} \Psi(1)^{\prime} C_{0} d \mathbf{W}(r) \\
& =\sigma^{2} \psi(1) \int_{0}^{1} \mathbf{W}^{*}(r)^{\prime} C_{0} d \mathbf{W}^{*}(r) \\
T^{-1} \sum_{t=1}^{N} \sum_{s=1-S}^{0} x_{S / 2, S t+s} \varepsilon S t+s & \stackrel{d}{\rightarrow} \frac{\sigma^{2}}{S} \int_{0}^{1} \mathbf{W}(r)^{\prime} \Psi(1)^{\prime} C_{S / 2} d \mathbf{W}(r) \\
& =\sigma^{2} \psi(-1) \int_{0}^{1} \mathbf{W}^{*}(r)^{\prime} C_{S / 2} d \mathbf{W}^{*}(r)
\end{aligned}
$$


and for $j=1, \ldots, S^{*}$,

$$
\begin{aligned}
T^{-1} \sum_{t=1}^{N} \sum_{s=1-S}^{0} x_{j, S t+s} \varepsilon_{S t+s} & \stackrel{d}{\rightarrow} \frac{\sigma^{2}}{S} \int_{0}^{1} \mathbf{W}(r)^{\prime} \mathbf{\Psi}(1)^{\prime} C_{j} d \mathbf{W}(r) \\
& =\frac{\sigma^{2} b_{j}}{2} \int_{0}^{1} \mathbf{W}^{\dagger}(r)^{\prime} C_{j} d \mathbf{W}^{\dagger}(r)-\frac{\sigma^{2} a_{j}}{2} \int_{0}^{1} \mathbf{W}^{\dagger}(r)^{\prime} C_{j}^{*} d \mathbf{W}^{\dagger}(r) \\
T^{-1} \sum_{t=1}^{N} \sum_{s=1-S}^{0} x_{j, S t+s}^{*} \varepsilon_{S t+s} & \stackrel{d}{\rightarrow} \frac{\sigma^{2}}{S} \int_{0}^{1} \mathbf{W}(r)^{\prime} \mathbf{\Psi}(1)^{\prime} C_{j}^{*} d \mathbf{W}(r) \\
& =\frac{\sigma^{2} a_{j}}{2} \int_{0}^{1} \mathbf{W}^{\dagger}(r)^{\prime} C_{j} d \mathbf{W}^{\dagger}(r)+\frac{\sigma^{2} b_{j}}{2} \int_{0}^{1} \mathbf{W}^{\dagger}(r)^{\prime} C_{j}^{*} d \mathbf{W}^{\dagger}(r) .
\end{aligned}
$$

Turning to the second term in the numerators of (A.28) and (A.29), using the results established in (A.15)-(A.18) we have that

$$
\sum_{t=1}^{N} \sum_{s=1-S}^{0} x_{0, S t+s}\left(e_{S t+s}^{k}-\varepsilon_{S t+s}\right)=\psi(1) \sum_{t=1}^{N} \sum_{s=1-S}^{0}\left(\sum_{j=1}^{(S t+s)-1} \varepsilon_{S t+s-j}\right) \sum_{i=k+1}^{\infty} \pi_{k, i} \varepsilon_{S t+s-i}
$$

and that

$$
\sum_{t=1}^{N} \sum_{s=1-S}^{0} x_{S / 2, S t+s}\left(e_{S t+s}^{k}-\varepsilon_{S t+s}\right)=\psi(-1) \sum_{t=1}^{N} \sum_{s=1-S}^{0}\left(\sum_{j=1}^{(S t+s)-1}(-1)^{j} \varepsilon_{S t+s-j}\right) \sum_{i=k+1}^{\infty} \pi_{k, i} \varepsilon_{S t+s-i}
$$

and that for $j=1, \ldots, S^{*}$,

$$
\begin{aligned}
\sum_{t=1}^{N} \sum_{s=1-S}^{0} x_{j, S t+s}\left(e_{S t+s}^{k}-\varepsilon_{S t+s}\right)= & b_{j} \sum_{t=1}^{N} \sum_{s=1-S}^{0}\left[\cos \left(\omega_{j}[s]\right)\left(\sum_{h=1}^{S} \cos \left(\omega_{j}[h-S]\right)\left[\sum_{g=1}^{t-1} \varepsilon_{S g-(s-h)}\right]\right)\right. \\
& \left.+\sin \left(\omega_{j}[s]\right)\left(\sum_{h=1}^{S} \sin \left(\omega_{j}[h-S]\right)\left[\sum_{g=1}^{t-1} \varepsilon_{S g-(s-h)}\right]\right)\right] \sum_{i=k+1}^{\infty} \pi_{k, i} \varepsilon_{S t+s-i} \\
& \left.+a_{j} \sum_{t=1}^{N} \sum_{s=1-S}^{0}\left[\sin \left(\omega_{j}[s]\right)\left(\sum_{h=1}^{S} \cos \left(\omega_{j}[h-S]\right)\right] \sum_{g=1}^{t-1} \varepsilon_{S g-(s-h)}\right]\right) \\
& \left.-\cos \left(\omega_{j}[s]\right)\left(\sum_{h=1}^{S} \sin \left(\omega_{j}[h-S]\right)\left[\sum_{g=1}^{t-1} \varepsilon_{S g-(s-h)}\right]\right)\right] \sum_{i=k+1}^{\infty} \pi_{k, i} \varepsilon_{S t+s-i} \\
\sum_{t=1}^{N} \sum_{s=1-S}^{*} x_{j, S t+s}^{*}\left(e_{S t+s}^{k}-\varepsilon_{S t+s}\right)= & b_{j} \sum_{t=1}^{N} \sum_{s=1-S}^{0}\left[\sin \left(\omega_{j}[s]\right)\left(\sum_{h=1}^{S} \cos \left(\omega_{j}[h-S]\right)\left[\sum_{g=1}^{t-1} \varepsilon_{S g-(s-h)}\right]\right)\right. \\
& \left.-\cos \left(\omega_{j}[s]\right)\left(\sum_{h=1}^{S} \sin \left(\omega_{j}[h-S]\right)\left[\sum_{g=1}^{t-1} \varepsilon_{S g-(s-h)}\right]\right)\right] \sum_{i=k+1}^{\infty} \pi_{k, i} \varepsilon_{S t+s-i} \\
& \left.-a_{j} \sum_{t=1}^{N} \sum_{s=1-S}^{0}\left[\sin \left(\omega_{j}[s]\right)\left(\sum_{h=1}^{S} \cos \left(\omega_{j}[h-S]\right)\right] \sum_{g=1}^{t-1} \varepsilon_{S g-(s-h)}\right]\right) \\
& \left.\left.+\sum_{h=1}^{S} \sin \left(\omega_{j}[h-S]\right)\left[\sum_{g=1}^{t-1} \varepsilon_{S g-(s-h)}\right]\right)\right] \sum_{i=k+1}^{\infty} \pi_{k, i} \varepsilon_{S t+s-i}
\end{aligned}
$$


where, in each case, as in Lemma 2 of Berk (1974), we have made use of the substitution $e_{S t+s}^{k}-\varepsilon_{S t+s}=$ $\sum_{i=k+1}^{\infty} d_{i} u_{S t+s-i}=\sum_{i=k+1}^{\infty} \pi_{k, i} \varepsilon_{S t+s-i}$, where $\sum_{i=k+1}^{\infty} \pi_{k, i}^{2} \leq \sum_{i=k+1}^{\infty} d_{i}^{2}=o\left(k^{-2 \tau}\right)$, and where $\tau$ is as defined in Assumption A.2. It is then straightforward to establish, paralleling the proof of part (a) of Lemma 3.1 in Chang and Park (2002, p.441), that $\sum_{t=1}^{N} \sum_{s=1-S}^{0} x_{j, S t+s}\left(e_{S t+s}^{k}-\varepsilon_{S t+s}\right)=o_{p}(T)$ for $j=0, \ldots,\lfloor S / 2\rfloor$ and that $\sum_{t=1}^{N} \sum_{s=1-S}^{0} x_{j, S t+s}^{*}\left(e_{S t+s}^{k}-\varepsilon_{S t+s}\right)=o_{p}(T)$ for $j=1, \ldots, S^{*}$. As an immediate consequence of these results, we then obtain, under Assumption A.2,

$$
\begin{aligned}
T^{-1} \sum_{t=1}^{N} \sum_{s=1-S}^{0} x_{j, S t+s} e_{S t+s}^{k} & =T^{-1} \sum_{t=1}^{N} \sum_{s=1-S}^{0} x_{j, S t+s} \varepsilon_{S t+s}+o_{p}(1), j=0, \ldots,\lfloor S / 2\rfloor \\
T^{-1} \sum_{t=1}^{N} \sum_{s=1-S}^{0} x_{j, S t+s}^{*} e_{S t+s}^{k} & =T^{-1} \sum_{t=1}^{N} \sum_{s=1-S}^{0} x_{j, S t+s}^{*} \varepsilon_{S t+s}+o_{p}(1), j=1, \ldots, S^{*}
\end{aligned}
$$

Combining the results in (A.31)-(A.36), and using applications of the CMT, we obtain the following:

$$
\begin{aligned}
T \widehat{\pi}_{0} & \stackrel{d}{\rightarrow} \frac{\int_{0}^{1} \mathbf{W}^{*}(r)^{\prime} C_{0} d \mathbf{W}^{*}(r)}{\psi(1) \int_{0}^{1} \mathbf{W}^{*}(r)^{\prime} C_{0} \mathbf{W}^{*}(r) d r} \\
T \widehat{\pi}_{S / 2} & \stackrel{d}{\rightarrow} \frac{\int_{0}^{1} \mathbf{W}^{*}(r)^{\prime} C_{S / 2} d \mathbf{W}^{*}(r)}{\psi(-1) \int_{0}^{1} \mathbf{W}^{*}(r)^{\prime} C_{S / 2} \mathbf{W}^{*}(r) d r}
\end{aligned}
$$

and for $j=1, \ldots, S^{*}$,

$$
\begin{aligned}
& T \widehat{\pi}_{j} \stackrel{d}{\rightarrow} \frac{b_{j} \int_{0}^{1} \mathbf{W}^{\dagger}(r)^{\prime} C_{j} d \mathbf{W}^{\dagger}(r)-a_{j} \int_{0}^{1} \mathbf{W}^{\dagger}(r)^{\prime} C_{j}^{*} d \mathbf{W}^{\dagger}(r)}{\frac{\left(a_{j}^{2}+b_{j}^{2}\right)}{2} \int_{0}^{1} \mathbf{W}^{\dagger}(r)^{\prime} C_{j} \mathbf{W}^{\dagger}(r) d r} \\
& T \widehat{\pi}_{j}^{*} \stackrel{d}{\rightarrow} \frac{a_{j} \int_{0}^{1} \mathbf{W}^{\dagger}(r)^{\prime} C_{j} d \mathbf{W}^{\dagger}(r)+b_{j} \int_{0}^{1} \mathbf{W}^{\dagger}(r)^{\prime} C_{j}^{*} d \mathbf{W}^{\dagger}(r)}{\frac{\left(a_{j}^{2}+b_{j}^{2}\right)}{2} \int_{0}^{1} \mathbf{W}^{\dagger}(r)^{\prime} C_{j} \mathbf{W}^{\dagger}(r) d r}
\end{aligned}
$$

Next observe that the corresponding $t$-statistics from (2.9) can be written as

$$
\begin{aligned}
t_{j} & =\hat{\sigma}^{-1} T \widehat{\pi}_{i} \times \sqrt{T^{-2} \sum_{t=1}^{N} \sum_{s=1-S}^{0}\left(x_{j, S t+s}\right)^{2}}+o_{p}(1), j=0, \ldots,\lfloor S / 2\rfloor \\
t_{h}^{*} & =\hat{\sigma}^{-1} T \widehat{\pi}_{h}^{*} \times \sqrt{T^{-2} \sum_{t=1}^{N} \sum_{s=1-S}^{0}\left(x_{h, S t+s}^{*}\right)^{2}}+o_{p}(1), h=1, \ldots, S^{*}
\end{aligned}
$$

where $\hat{\sigma}^{2}$ is the usual OLS variance estimator from (2.9); that is, $\hat{\sigma}^{2}:=T^{-1} \sum_{t=1}^{N} \sum_{s=1-S}^{0}\left(\hat{e}_{S t+s}^{k}\right)^{2}=$ $T^{-1}\left(\mathbf{u}^{\prime} \mathbf{u}-\mathbf{u}^{\prime} \mathbf{Z}_{k}\left(\mathbf{Z}_{k}^{\prime} \mathbf{Z}_{k}\right)^{-1} \mathbf{Z}_{k}^{\prime} \mathbf{u}\right)$. It then follows immediately from parts (i) and (ii) of Lemma A.1 that $\hat{\sigma}^{2}=T^{-1} \mathbf{u}^{\prime} \mathbf{u}+o_{p}(1)$. Then since part (c) of Lemma 3.1 in Chang and Park (2002) also applies here, we obtain the result that $\hat{\sigma}^{2} \stackrel{p}{\rightarrow} \sigma^{2}$. Substituting this result together with those in (A.38)-(A.41) into (A.42)-(A.43) and using applications of the CMT we then, after some simple manipulations, finally obtain the stated results in Proposition 1, where we have defined the independent standard Brownian motions, $W_{i}(r):=\mathbf{v}_{i}^{\prime} \mathbf{W}^{*}(r), i=0, S / 2, W_{j}(r):=\mathbf{c}_{j}^{\prime} \mathbf{W}^{\dagger}(r)$ and $W_{j}^{*}(r):=\mathbf{c}_{j}^{* \prime} \mathbf{W}^{\dagger}(r)^{\prime}$, where $\mathbf{c}_{j}^{\prime}$ and $\mathbf{c}_{j}^{* \prime}$ are the first rows of $\mathbf{v}_{j}^{\prime}$ and $\mathbf{v}_{j}^{* \prime}$, respectively for $j=1, \ldots, S^{*}$. 